$$
\text { Pontifícia Universidade C Católica }
$$

João Vieira de Araújo

Metodologia para determinação do mercado potencial de
um sistema de cogeração com microturbina para produção
de energia elétrica e água quente

Dissertação de Mestrado

Dissertação apresentada como requisito parcial para obtenção do título de Mestre pelo Programa de PósGraduação em Metrologia da PUC-Rio. Área de Concentração: Metrologia para Qualidade e Inovação.

Orientador: Prof. Alcir de Faro Orlando 


$$
\text { Pontifícia Universidade } \text { Católica }_{\text {do Rio de Janeiro }}
$$

João Vieira de Araújo

\section{Metodologia para determinação do mercado potencial de um sistema de cogeração com microturbina para produção de energia elétrica e água quente}

Dissertação apresentada como requisito parcial para obtenção do grau de Mestre pelo Programa de Pós-Graduação em Metrologia do Centro Técnico Científico da PUC-Rio. Aprovada pela Comissão Examinadora e homologada pela Coordenação Setorial de PósGraduação, formalizado pelas respectivas assinaturas.

Comissão Examinadora:

Prof. Dr. Alcir de Faro Orlando Orientador

Departamento de Engenharia Mecânica Programa de Pós-Graduação em Metrologia (PósMQI) Pontifícia Universidade Católica do Rio de Janeiro (PUC-Rio)

Prof. Dr. José Alberto dos Reis Parise

Departamento de Engenharia Mecânica Pontifícia Universidade Católica do Rio de Janeiro (PUC-Rio)

Prof. Dr. Eloy Fernandez Y Fernandez

Departamento de Engenharia Mecânica Pontifícia Universidade Católica do Rio de Janeiro (PUC-Rio)

Prof. Dr. Carlos Eduardo Reuther de Siqueira PETROBRAS - Petróleo Brasileiro S.A

Coordenação Setorial de Pós-Graduação:

Prof. José Eugênio Leal Coordenador Setorial de Pós-Graduação do Centro Técnico Científico (PUC-Rio)

Rio de Janeiro, 20 de abril de 2007. 
Todos os direitos reservados. É proibida a reprodução total ou parcial do trabalho sem autorização da universidade, do autor e do orientador.

\section{João Vieira de Araújo}

Graduado em Engenharia Elétrica pela Universidade Gama Filho, em 1988, MBA em Administração de Negócios pelo IBMEC Business School em 2000, MBA em Energia Elétrica pelo IBMEC Business School em 2002 e MBA em Gerência de Projetos pela Fundação Getúlio Vargas em 2003.

Ficha Catalográfica

Araújo, João Vieira de

Metodologia para determinação do mercado potencial de um sistema de cogeração com microturbina para produção de energia elétrica e água quente / João Vieira de Araújo ; orientador: Alcir de Faro Orlando. - 2007.

255 f. ; $30 \mathrm{~cm}$

Dissertação (Mestrado em Metrologia, Qualidade e Inovação) - Pontifícia Universidade Católica do Rio de Janeiro, Rio de Janeiro, 2007.

Inclui bibliografia

1. Metrologia - Teses. 2. Cogeração. 3. Microturbina. 4. Desempenho. 5. Geração distribuída. 6. Incertezas. I. Orlando, Alcir de Faro. II. Pontifícia Universidade Católica do Rio de Janeiro. Programa de PósGraduação em Metrologia para a Qualidade e Inovação. III. Título.

CDD: 389.1 

aos meus filhos Mônica e João Luis. 


\section{Agradecimentos}

Ao Professor Alcir do Faro Orlando, pela sua constante e valorosa orientação e dedicação, não só para desenvolvimento deste trabalho, como também para meu aperfeiçoamento profissional.

Ao Professor Maurício Nogueira Frota, pelo seu constante apoio ao longo do curso e em reconhecimento ao seu trabalho de divulgação dos benefícios da metrologia para a sociedade.

Aos engenheiros e pesquisadores Luiz Gustavo do Val e Marlon Huamani Bellido do Laboratório de Avaliação Metrológica e Energética - LAME da PUC-Rio pela ajuda na realização das análises dos dados coletados e pela dedicação, apoio e enriquecimento deste projeto.

Aos professores José Antônio Pimenta-Bueno, Antonio José Junqueira Botelho e Sérgio Yates, pelo estímulo ao empreendedorismo e à inovação.

Aos Professores Glauter Rocha, Paula Medeiros Proença Gouvêa e Leonardo Macrini pelo dinamismo de suas aulas, empenho e atenção.

A LIGHT Energia pela oportunidade de desenvolvimento e incentivo ao crescimento profissional.

Ao Amigo José Tenório Barreto Júnior por todo o incentivo para a realização do mestrado no PósMQI.

Ao Programa de Pesquisa e Desenvolvimento do Setor Elétrico, que em observância à Lei $n^{\circ}$ 9991, de 24/01/2000, e à resolução ANEEL $n^{\circ} 271$ de 19/07/2000, financiou o projeto de P\&D 035/2004 - Avaliação de desempenho de planta de produção de energia elétrica.

A Deus pelo amparo e oportunidades. 


\section{Resumo}

Araújo, João Vieira de; Orlando, Alcir de Faro (Orientador). Metodologia para determinação do mercado potencial de um sistema de cogeração com microturbina para produção de energia elétrica e água quente. Rio de Janeiro, 2007. 255p. Dissertação de Mestrado - Programa de Pós-Graduação em Metrologia, Qualidade e Inovação (PósMQI), Pontifícia Universidade Católica do Rio de Janeiro.

O uso seqüencial de energia elétrica e energia térmica a partir da energia química do mesmo combustível tem sido estudado para melhorar o custo benefício de uma geração distribuída. O objetivo deste estudo é determinar o mercado potencial desta tecnologia na região de influência da concessionária local de energia elétrica. Para alcançar este objetivo, foi necessário avaliar o desempenho de um sistema de cogeração com uma microturbina fornecendo potência elétrica e energia térmica através dos gases de exaustão que aquecem água para o ginásio esportivo da PUC-Rio. O sistema está configurado por uma microturbina, uma caldeira de recuperação, um reservatório térmico (boiler) e uma caixa para alimentação de água fria. Resultados obtidos de testes de curta duração, através de uma metodologia desenvolvida, para a avaliação do desempenho do sistema de cogeração na produção de energia elétrica e térmica, são apresentados. Os testes foram realizados para potências elétricas de 100, 75, 50 e $25 \%$ da carga máxima da microturbina. Em adição, testes para avaliação do desempenho ao longo do tempo em função das variações da carga e das condições ambientais foram estudados. Além disso, através de uma metodologia desenvolvida, mostram-se os resultados nos cálculos de incerteza dos parâmetros analisados. Dados de consumo de energia elétrica em janeiro de 2003, de cerca de 8000 consumidores na área de concessão da LIGHT SESA, em diferentes classes tarifárias, atualizadas para 2007, foram utilizados com a metodologia desenvolvida para estimar o mercado potencial de energia elétrica na região de influência da concessionária local de energia elétrica em 384,5 MW e $20415 \mathrm{MWh} / \mathrm{mês,}$ considerando uma taxa interna de retorno mínimo de $15 \%$ a.a. Foi mostrado que o elevado custo da microturbina é uma barreira para sua introdução no mercado. Entretanto, a cogeração pode colaborar substancialmente para melhorar o custo benefício. Estas conclusões foram obtidas a partir do tempo de retorno do investimento no sistema pelos consumidores analisados.

\section{Palavras-chave}

Metrologia; Cogeração; Microturbina; Desempenho; Geração distribuída; Incertezas. 


\section{Abstract}

Araujo, João Vieira de; Orlando, Alcir de Faro (Advisor). Methodology for determining the potential market of a microturbine based cogeneration system for electric energy and hot water production. Rio de Janeiro, 2007. 255p. MSc. Dissertation - Programa de Pós-Graduação em Metrologia, Qualidade e Inovação (PósMQI), Pontifícia Universidade Católica do Rio de Janeiro.

The sequential use of electric energy and thermal energy from the chemical energy of the same fuel has been studied to improve the cost benefit of a distributed generation. The objective of this study is to evaluate the potential market of this technology in the region of influence of the local utility of electric energy.utility company (LIGHT). In order to attain this objective, it was necessary to evaluate the performance of a micro turbine based cogeneration system used for supplying electrical and thermal energy to PUC-RIO'S Gymnasium. The system consists of a microturbine, a heat recovery boiler, and a cold water feeding reservoir. The short duration test results are presented for the evaluation of the cogeneration system performance for the production of electric and thermal energy. The tests were carried on for $100,75,50$ and $25 \%$ micro turbine electric energy generation loads. Time performance of the micro turbine was also studied. Moreover, a methodology was shown to calculate the uncertainty of measurements of several performance parameters. The micoturbine marked potential was estimated by analyzing the consumption of about 8000 clients in January 2003, updating the tariffs to 2007 , resulting in $384,5 \mathrm{MW}$ and $20415 \mathrm{MWh} /$ month, considering a minimum internal rate of return of investiment of $15 \%$ per year, during a 15 year period. It was concluded that the microturbine cost is still a barrier to its market penetration. Cogeneration can be used to improve the cost benefit of the system.

\section{Keywords}

Metrology; Cogeneration; Microturbine; Performance; Distributed generation; Uncertainties. 


\section{Sumário}

1 Introdução 22

1.1. Considerações iniciais 22

1.2. Estado da arte 23

1.3. Objetivo 25

1.4. Estruturação 25

2 Fundamentos Teóricos $\quad 27$

2.1. Geração distribuída e cogeração $\quad 27$

2.2. A Cogeração a gás natural 31

2.3. Caldeiras de recuperação 33

2.4. Microturbinas a gás (MTG) 34

2.4.1. Aplicações $\quad 35$

2.4.2. Descrição tecnológica 37

2.4.2.1. Princípio de funcionamento 37

2.4.2.2. Componentes básicos 39

2.4.2.3. Operação com sistemas de cogeração 42

2.4.2.4. Aspectos técnicos - econômicos e ambientais 43

2.4.2.5. Barreiras tecnológicas e de penetração no mercado 48

2.5. Reservatório Térmico (Boiler) 50

3 Procedimento Experimental - Descrição do Sistema 52

3.1. Utilização do combustível

3.2. Geração de energia $\quad 59$

3.3. Aproveitamento da energia térmica do sistema 68

3.4. Instrumentação $\quad 75$

3.4.1. Descrição dos sensores $\quad 77$

3.4.2. Medidores de vazão tipo turbina 78

3.4.3. Termômetro de resistência tipo PT-100 81

3.4.4. Transmissor de pressão $\quad 83$

3.4.5. Aquisitor de dados $\quad 84$

4 Comissionamento da Microturbina em Carga Máxima 85

4.1. Potência de saída e rendimento térmico obtido através de testes feitos com o fabricante utilizando o software disponível pela microturbina $\quad 85$

$\begin{array}{ll}\text { 4.1.1. Metodologia } & 94\end{array}$

4.1.2. Análise 99

4.2. Tensão e freqüência elétrica obtida através dos testes feitos com o fabricante 102

4.2.1. Metodologia 103

$\begin{array}{ll}\text { 4.2.2. Análise } & 110\end{array}$

5 Resultados 115

5.1. Desempenho na produção de energia elétrica e térmica do sistema de cogeração em carga máxima $\quad 115$

5.1.1. Metodologia 115

5.1.2. Análise 122

5.2. Critérios de avaliação e desempenho na produção e qualidade de energia elétrica e térmica com diferentes cargas de operação $\quad 127$

$\begin{array}{ll}\text { 5.2.1. Metodologia } & 128\end{array}$

5.2.2. Desempenho na produção de calor e potência 130 
5.2.3. Desempenho operacional 134

5.2.4. Cálculo das incertezas 135

5.2.5. Análise 140

5.3. Critérios de avaliação e desempenho parcial da planta de cogeração, após o reparo do mecanismo de acionamento do damper da caldeira de recuperação

5.3.1. Resultados e análise 153

5.3.1.1. Resultados 153

5.3.1.2. Análise 167

5.4. Desempenho da planta de cogeração em função das variações de carga e das condições ambientais $\quad 170$

5.4.1. Testes realizados 170

5.4.1.1. Resultados 171

5.4.1.2. Análise 173

5.5. Resumo das avaliações de desempenho e falhas na planta de cogeração

5.5.1. Resumo das avaliações de desempenho 176

$\begin{array}{ll}\text { 5.5.2. Falhas e soluções } & 179\end{array}$

6 Avaliação da Penetração da Microturbina no Mercado da LIGHT 182

6.1. Introdução 182

6.2. Definições e fundamentos teóricos 184

6.3. Clientes potenciais para autoprodução de energia 186

6.4. Custo atual do fornecimento de energia elétrica pela concessionária 187

6.5. Custo do investimento na microturbina e no geradores para autoprodução de energia elétrica 187

6.6. Custo de operação e manutenção da microturbina e dos geradores 188

6.7. Custo do combustível para operação da microturbina e dos geradores 188

6.8. Tempo de retorno do investimento e Payback

6.9. Número de clientes com Payback igual ou inferior a 5,8 anos 190

6.10. Estimativa do mercado deslocado da Light 193

6.11. Conclusões 194

7 Conclusões e Recomendações 196

8 Referências bibliográficas 199

Apêndices 205

Apêndice A - Teste realizado no dia 09 de Junho de 2006

Apêndice B - Teste realizado no dia 12 de Junho de 2006

Apêndice C - Teste realizado no dia 20 de Junho de 2006

Apêndice D - Teste realizado no dia 21 de Junho de 2006

Apêndice E - Teste realizado no dia 23 de Junho de 2006

Apêndice F - Teste realizado no dia 29 de Junho de 2006 


\section{Lista de figuras}

Figura 1 - Aplicações Típicas de Cogeração. 28

Figura 2 - Diagrama esquemático de um sistema de cogeração. 30

Figura 3 - Esquema da caldeira de recuperação. 34

Figura 4 - Microturbina com recuperador de calor. 38

Figura 5 - Fotografia do conjunto compressor e turbina da microturbina Capstone.

Figura 6 - Microturbinas Capstone - Modelo 330 (30 kW) - 10 unidades. $\quad 39$

Figura 7 - Desempenho de uma microturbina de $60 \mathrm{~kW}$ em função da temperatura ambiente.

Figura 8 - Desempenho em carga parcial de uma microturbina de $30 \mathrm{Kw.} 45$

Figura 9 - Emissões de uma microturbina de $30 \mathrm{~kW}$ como função da carga.

Figura 10 - Esquema do Tanque do Reservatório Térmico.

Figura 11 - Linha de alimentação de Gás Natural da planta de cogeração instalada na PUC-Rio.

Figura 12 - Estação de medição de Gás Natural da planta de cogeração da PUC-Rio.

Figura 13 - Linha de alimentação de Gás Natural da planta de cogeração instalada na PUC-Rio.

Figura 14 - Sistema de fornecimento de gás natural e medição da microturbina, antes da instalação da medição de vazão.

Figura 15 - Sistema de combustível da microturbina Capstone C30 LPNG (a).

Figura 16 - Sistema de combustível da microturbina Capstone C30 LPNG (b).

Figura 17 - Diagrama de Bloco do funcionamento do sistema de combustível da microturbina Capstone C30 LPNG.

Figura 18 - Injetor de combustível da microturbina Capstone C30 LPNG. $\quad 59$

Figura 19 - Microturbina Capstone C30 LPNG.

Figura 20 - Esquema de funcionamento do sistema eletrônico de potência da microturbina Capstone C30 LPNG.

Figura 21 - Sistema de acionamento e proteção da microturbina Capstone C30 LPNG na entrada da rede elétrica da PUCRio.

Figura 22 - Painel de controle da microturbina Capstone C30 LPNG.

Figura 23 - Interface de comunicação da microturbina Capstone C30 LPNG.

Figura 24 - Modo de operação da microturbina instalada na PUC-Rio (Carga Base).

Figura 25 - Ligação elétrica gerador tipo microturbina Capstone C30 LPNG ao barramento da PUC-Rio.

Figura 26 - Autotransformador Trifásico de 45 kVA instalado na PUCRio.

Figura 27 - Características dos gases de exaustão da Microturbina Capstone C30 Recuperated LPNG.

Figura 28 - Instalação do Duto de Exaustão entre a Microturbina Capstone C30 LPNG e a Caldeira de Recuperação ITC1.

Figura 29 - Detalhe da Caldeira de Recuperação ITC1 da planta de cogeração instalada na PUC-Rio.

Figura 30 - Painel do Controlador de Temperatura. 
Figura 31 - Reservatório Térmico (Boiler) da planta de cogeração da PUC-Rio.

Figura 32 - Boiler e Caixa de Água da planta de cogeração da PUC-Rio. 73

Figura 33 - Misturadores de água quente nos banheiros do ginásio da PUC-Rio.

Figura 34 - Instalação da microturbina Capstone C30 LPNG - Caldeira de Recuperação ITC1.

Figura 36 - Esquema do Sistema de Medição da Bancada da Microturbina a Gás.

Figura 37 - Esquema de ligação dos sensores ao sistema de aquisição de dados.

Figura 38 - Curva do Sensor de Vazão de Gás Natural tipo Turbina.

Figura 39 - Esquema do cavalete de medição para o Gás Natural.

Figura 40 - Curva do Sensor de Vazão de Água.

Figura 41 - Potência e eficiência elétrica versus temperatura ambiente na pressão atmosférica de $101,325 \mathrm{kPa}$ Modelo C30 (recuperated) LPNG.

Figura 42 - Temperatura e vazão mássica dos gases de exaustão versus temperatura ambiente na pressão atmosférica de 101,325 $\mathrm{kPa}$ - Modelo C30 (recuperated) LPNG.

Figura 43 - Power Ratio versus pressão atmosférica - Modelo C30 (recuperated) LPNG.

Figura 44 - "E.B.P.P.R." e "E.B.P.E.R." versus a perda de carga na exaustão - Modelo C30 (recuperated) LPNG.

Figura 45 - Tolerância permitida e faixa de variação dos valores registrados das freqüências elétricas durante o desenvolvimento do teste da microturbina Capstone.

Figura 46 - Valores das tensões da saída na fase A (V) da microturbina Capstone durante o desenvolvimento do teste. 113

Figura 47 - Valores das tensões da saída na fase B (V) da microturbina Capstone durante o desenvolvimento do teste. 113

Figura 48 - Valores das tensões da saída na fase C (V) da microturbina Capstone durante o desenvolvimento do teste. 114

Figura 49 - Variação das Temperaturas da água de entrada (TÁGUA IN) e de saída (TÁGUA OUT) da caldeira de recuperação durante o desenvolvimento do teste no Sistema de Cogeração da microturbina Capstone C30 LPNG.

Figura 50 - Variação da Temperatura dos gases de exaustão de entrada à caldeira (TEX IN) e de saída da caldeira (TEX OUT) durante o desenvolvimento do teste no Sistema de Cogeração.

Figura 51 - Variação da Potência da microturbina com respeito à Temperatura de Entrada do Ar durante o desenvolvimento do teste no Sistema de Cogeração.

Figura 52 - Variação do Calor dos Gases na Caldeira (QCALD) e o Calor absorvido pela água (QAGUA) durante o desenvolvimento do teste de Sistema de Cogeração.

Figura 53 - Variação da RPM durante o desenvolvimento do teste realizado no dia 27/05/2006.

Figura 54 - Variação da Freqüência durante o desenvolvimento do teste realizado no dia 27/05/2006.. 
Figura 55 - Variação da Potência durante o desenvolvimento do teste realizado no dia 27/05/2006.

Figura 56 - Variação da Tensão Fase _ A durante o desenvolvimento do teste realizado no dia 27/05/2006.

Figura 57 - Variação da Tensão Fase _ B durante o desenvolvimento do teste realizado no dia 27/05/2006.

Figura 58 - Variação da Tensão Fase _ C durante o desenvolvimento do teste realizado no dia 27/05/2006.

Figura 59 - Variação da RPM e Potência durante a partida da microturbina no teste realizado no dia 27/05/2006.

Figura 60 - Variação da Temperatura de Exaustão durante a partida da microturbina no teste realizado no dia 27/05/2006.

Figura 61 - Variação da Tensão durante a partida da microturbina no teste realizado no dia 27/05/2006.

Figura 62 - Variação da Corrente durante a partida da microturbina no teste realizado no dia 27/05/2006.

Figura 63 - Variação da RPM durante o desenvolvimento do teste no dia 27/05/2006.

Figura 64 - Variação da Freqüência durante o desenvolvimento do teste no dia 27/05/2006.

Figura 65 - Variação da Potência durante o desenvolvimento do teste no dia 27/05/2006.

Figura 66 - Variação da Tensão na Fase _ A durante o desenvolvimento do teste no dia 27/05/2006.

Figura 67 - Variação da Tensão na Fase _ B durante o desenvolvimento do teste no dia 27/05/2006.

Figura 68 - Variação da Tensão na Fase _ C durante o desenvolvimento do teste no dia 27/05/2006.

Figura 69 - Variação da RPM e Potência durante a partida da microturbina no dia 27/05/2006.

Figura 70 - Variação da Temperatura de Exaustão durante a partida da microturbina no dia 27/05/2006.

Figura 71 - Variação da Tensão durante a partida da microturbina no dia 27/05/2006.

Figura 72 - Variação da Corrente durante a partida da microturbina no dia 27/05/2006.

Figura A.1 - Variação da Temperatura de saída da água da caldeira durante o desenvolvimento do teste.

Figura A.2 - Variação da Temperatura dos gases de exaustão que entram (T EX IN) e saem (T EX OUT) da caldeira de recuperação durante o desenvolvimento do teste.

Figura A.3 - Variação das RPM e Potência Gerada pelo sistema (Po) durante o desenvolvimento do teste.

Figura A.4 - Variação das RPM do compressor de Gás Natural durante o desenvolvimento do teste.

Figura A.5 - Variação da eficiência elétrica, térmica e total da planta de cogeração durante o desenvolvimento do teste.

Figura A.6 - Potência elétrica gerada (Po) e Energia fornecida pelo Gás Natural $(\mathrm{HI})$ da planta de cogeração durante o desenvolvimento do teste.

Figura A.7 - Variação da Temperatura de exaustão da microturbina durante o desenvolvimento do teste.

Figura A.8 - Variação do calor da água (Q ÁGUA) e o calor dos gases de exaustão (Q GASES EXAUSTÃO) durante o desenvolvimento do teste. 
Figura B.1 - Variação da Temperatura de saída da água da caldeira durante o desenvolvimento do teste.

Figura B.2 - Variação da Temperatura dos gases de exaustão que entram (T EX IN) e saem (T EX OUT) da caldeira de recuperação durante o desenvolvimento do teste.

Figura B.3 - Variação das RPM e Potência Gerada pelo sistema (Po) durante o desenvolvimento do teste.

Figura B.4 - Variação das RPM do compressor de Gás Natural durante o desenvolvimento do teste.

Figura B.5 - Variação da eficiência elétrica, térmica e total da planta de cogeração durante o desenvolvimento do teste.

Figura B.6 - Potência elétrica gerada $(\mathrm{Po})$ e Energia fornecida pelo Gás Natural $(\mathrm{HI})$ da planta de cogeração durante 0 desenvolvimento do teste.

Figura B.7 - Variação da Temperatura de exaustão de microturbina durante o desenvolvimento do teste.

Figura B.8 - Variação do calor da água (Q ÁGUA) e o calor dos gases de exaustão (Q GASES EXAUSTÃO) durante o desenvolvimento do teste.

Figura C.1 - Variação da Temperatura de saída da água da caldeira durante o desenvolvimento do teste.

Figura C.2 - Variação da Temperatura dos gases de exaustão que entram (T EX IN) e saem (T EX OUT) da caldeira de recuperação durante o desenvolvimento do teste.

Figura C.3 - Variação das RPM e Potência Gerada pelo sistema (Po) durante o desenvolvimento do teste.

Figura C.4 - Variação das RPM do compressor de Gás Natural durante o desenvolvimento do teste.

Figura C.5 - Variação da eficiência elétrica, térmica e total da planta de cogeração durante o desenvolvimento do teste.

Figura C.6 - Potência elétrica gerada (Po) e Energia fornecida pelo Gás Natural $(\mathrm{HI})$ da planta de cogeração durante o desenvolvimento do teste.

Figura C.7 - Variação da Temperatura de exaustão de microturbina durante o desenvolvimento do teste.

Figura C.8 - Variação do calor da água (Q ÁGUA) e o calor dos gases de exaustão (Q GASES EXAUSTÃO) durante o desenvolvimento do teste.

Figura D.1 - Variação da Temperatura de saída da água da caldeira durante o desenvolvimento do teste.

Figura D.2 - Variação da Temperatura dos gases de exaustão que entram (T EX IN) e saem (T EX OUT) da caldeira de recuperação durante o desenvolvimento do teste.

Figura D.3 - Variação das RPM e Potência Gerada pelo sistema (Po) durante o desenvolvimento do teste.

Figura D.4 - Variação das RPM do compressor de Gás Natural durante o desenvolvimento do teste.

Figura D.5 - Variação da eficiência elétrica, térmica e total da planta de cogeração durante o desenvolvimento do teste.

Figura D.6 - Potência elétrica gerada (Po) e Energia fornecida pelo Gás Natural $(\mathrm{HI})$ da planta de cogeração durante o desenvolvimento do teste.

Figura D.7 - Variação da Temperatura de exaustão de microturbina durante o desenvolvimento do teste. 
Figura D.8 - Variação do calor da água (Q ÁGUA) e o calor dos gases de exaustão (Q GASES EXAUSTÃO) durante o desenvolvimento do teste.

Figura E.1 - Variação da Temperatura de saída da água da caldeira durante o desenvolvimento do teste.

Figura E.2 - Variação da Temperatura dos gases de exaustão que entram (T EX IN) e saem (T EX OUT) da caldeira de recuperação durante o desenvolvimento do teste.

Figura E.3 - Variação das RPM e Potência Gerada pelo sistema (Po) durante o desenvolvimento do teste.

Figura E.4 - Variação das RPM do compressor de Gás Natural durante o desenvolvimento do teste.

Figura E.5 - Variação da eficiência elétrica, térmica e total da planta de cogeração durante o desenvolvimento do teste.

Figura E.6 - Potência elétrica gerada (Po) e Energia fornecida pelo Gás Natural (HI) da planta de cogeração durante o desenvolvimento do teste.

Figura E.7 - Variação da Temperatura de exaustão de microturbina durante o desenvolvimento do teste.

Figura E.8 - Variação do calor da água (Q ÁGUA) e o calor dos gases de exaustão (Q GASES EXAUSTÃO) durante o desenvolvimento do teste.

Figura F.1 - Variação da Temperatura de saída da água da caldeira durante o desenvolvimento do teste.

Figura F.2 - Variação da Temperatura dos gases de exaustão que entram (T EX IN) e saem (T EX OUT) da caldeira de recuperação durante o desenvolvimento do teste.

Figura F.3 - Variação das RPM e Potência Gerada pelo sistema (Po) durante o desenvolvimento do teste.

Figura F.4 - Variação das RPM do compressor de Gás Natural durante o desenvolvimento do teste.

Figura F.5 - Variação da eficiência elétrica, térmica e total da planta de cogeração durante o desenvolvimento do teste.

Figura F.6 - Potência elétrica gerada (Po) e Energia fornecida pelo Gás Natural $(\mathrm{HI})$ da planta de cogeração durante o desenvolvimento do teste.

Figura F.7 - Variação da Temperatura de exaustão de microturbina durante o desenvolvimento do teste.

Figura F.8 - Variação do calor da água (Q ÁGUA) e o calor dos gases de exaustão (Q GASES EXAUSTÃO) durante o desenvolvimento do teste. 


\section{Lista de tabelas}

Tabela 1 - Características de desempenho das microturbinas.

Tabela 2 - Estimativa do custo de capital de microturbinas operando em paralelo com a rede da concessionária.

Tabela 3 - Estimativa do custo de capital de sistemas de cogeração com microturbinas operando em paralelo com a rede da concessionária.

Tabela 4 - Substituição do compressor a diversas pressões do combustível.

Tabela 5 - Características Elétricas da microturbina Capstone modelo C30 operada em paralelo à rede elétrica local.

Tabela 6 - Desempenho da Microturbina C30 (recuperated) LPNG.

Tabela 7 - Parâmetros de desempenho como função da temp. amb. e pressão atm. de 101,325 kPa - Modelo C30 (recuperated) LPNG - Dados do fabricante.

Tabela 8 - Fator de degradação da potência elétrica como função da pressão atmosférica - Modelo C30 (recuperated) LPNG Dados do fabricante.

Tabela 9 - Fator de degradação da potência e eficiência elétrica como função da perda de carga dos gases de exaustão - Modelo C30 (recuperated) LPNG - dados do fabricante.

Tabela 10 - Dados do teste de comissionamento da microturbina realizado pelo representante da Capstone em 18/08/05.

Tabela 11 - Características do gás natural do gás natural fornecido pela CEG em 18/08/2005 - média diária.

Tabela 12 - Valores calculados de parâmetros de desempenho da Microturbina.

Tabela 13 - Valor calculado de potencial máximo de cogeração da planta da PUC-Rio

Tabela 14 - Dados elétricos do teste realizado na microturbina, na PUCRio.

Tabela 15 - Exatidão dos sensores internos da microturbina Capstone modelo C30 (Recuperated) LPNG, de $28 \mathrm{KW}$.

Tabela 16 - Resumo dos resultados de avaliação da freqüência elétrica durante o desenvolvimento do teste.

Tabela 17 - Resumo dos resultados de avaliação das tensões para cada fase durante o desenvolvimento do teste.

Tabela 18 - Variação máxima dos valores registrados das tensões para cada fase durante o desenvolvimento do teste.

Tabela 19 - Valores médios das potências no cálculo da eficiência do sistema eletrônico de potência da microturbina.

Tabela 20 - Cálculo dos valores da potência média gerada pela microturbina baseado nas potências médias em cada fase.

Tabela 21 - Características médias do Gás Natural fornecido pela CEG em 25/01/2006.

Tabela 22 - Valores das constantes da Equação de Callendar - Van Dusen.

Tabela 23 - Dados do teste do Sistema de Cogeração na PUC-Rio, realizados no dia 25/01/2006. 
Tabela 24 - Desempenho do Sistema de Cogeração calculado com os dados do teste realizado no 25/01/2006.

Tabela 25 - Desempenho do Sistema de Cogeração estimado a partir do procedimento da Capstone, para as condições ambientais do local de operação.

Tabela 26 - Testes controlados da planta de cogeração do ginásio esportivo da PUC-Rio.

Tabela 27 - Variação permitida das condições de operação.

Tabela 28 - Divisores para distribuição de probabilidade $(95,45 \%$ de nível de confiança).

Tabela 29 - Características médias do Gás Natural fornecido pela CEG em 27/05/2006.

Tabela 30 - Variabilidade observada nos parâmetros operacionais no teste realizado no dia 27/05/2006.

Tabela 31 - Valores médios, máximos, mínimos e desvio padrão da Freqüência e Tensão Elétrica durante o desenvolvimento do teste no teste realizado no dia 27/05/2006.

Tabela 32 - Desempenho na produção de Potência e Calor no teste realizado no dia 27/05/2006.

Tabela 33 - Condições do GN, fluido recuperador de calor (água) e gases de exaustão da planta de cogeração da PUC-Rio.

Tabela 34 - Resumo das incertezas dos instrumentos de medição e dos parâmetros medidos, para a máxima potência, no dia do teste (27/05/2006), na planta de cogeração da PUC-Rio.

Tabela 35 - Testes controlados da planta de cogeração do ginásio esportivo da PUC-Rio, no dia 09/06/06.

Tabela 36 - Características médias do Gás Natural fornecido pela CEG em 09/06/2006.

Tabela 37 - Variação permitida das condições de operação.

Tabela 38 - Variabilidade observada nos parâmetros operacionais no teste realizado o dia 09/06/2006.

Tabela 39 - Valores médios, máximos, mínimos e desvio padrão da Freqüência e Tensão Elétrica durante o desenvolvimento do teste no dia 27/05/2006.

Tabela 40 - Desempenho na produção de Potência e Calor durante o desenvolvimento do teste no dia 27/05/2006.

Tabela 41 - Condições do GN, fluido recuperador de calor (água) e gases de exaustão da planta de cogeração da PUC-Rio

Tabela 42 - Resumo das incertezas dos instrumentos de medição e dos parâmetros medidos, para a máxima potência, no dia do teste (27/05/2006), na planta de cogeração da PUC-Rio.

Tabela 43 - Valores da relação ar - combustível, vazão de ar, vazão de combustível e vazão dos gases de exaustão no dia do teste.

Tabela 44 - Valores calor da água e calor dos gases de exaustão no dia 27/05/2006.

Tabela 45 - Características médias do Gás Natural fornecido pela CEG, para o mês de Junho de 2006.

Tabela 46 - Características dos testes realizados na planta de cogeração da PUC-Rio.

Tabela 47 - Valores médios dos parâmetros principais nos testes realizados na planta de cogeração da PUC-Rio.

Tabela 48 - Comparação da potência fornecida e eficiência calculada nos testes realizados na planta de cogeração da PUC-Rio e os fornecidos pelo fabricante. 
Tabela 49 - Potência indicada no painel da microturbina a gás e potência média gerada.

Tabela 50 - Potência e eficiência elétrica da microturbina a gás (fabricante e condições da planta).

Tabela 51 - Tarifa convencional da concessionária

Tabela 52 - Tarifa horo-sazonal azul da concessionária.

Tabela 53 - Tarifa horo-sazonal verde da concessionária. 183

Tabela 54 - Número de clientes por grupo tarifário e tarifa. 186

Tabela 55 - Número de clientes por grupo tarifário e classe. 186

Tabela 56 - Número de clientes por tarifa e classe. 186

Tabela 57 - Tarifa de GN por faixa de consumo em 2007.

Tabela 58 - Relação entre Payback e TIR. 190

Tabela 59 - Número de clientes por tarifa e horário com Payback em anos para a microturbina, considerando um consumo específico de $0,636 \mathrm{~N} \mathrm{~m}^{3} / \mathrm{kWh}$.

Tabela 60 - Número de clientes, com TIR maior ou igual a 15\%, por grupo tarifário e tarifa, considerando a microturbina com o consumo específico de $0,636 \mathrm{~N} \mathrm{~m}^{3} / \mathrm{kWh}$.

Tabela 61 - Número de clientes por tarifa e horário com Payback em anos, considerando a microturbina com o consumo específico de $0,440 \mathrm{~N} \mathrm{~m}^{3} / \mathrm{kWh}$.

Tabela 62 - Número de clientes, com TIR maior ou igual a 15\%, por grupo tarifário e tarifa, considerando a microturbina com o consumo específico de $0,440 \mathrm{~N} \mathrm{~m}^{3} / \mathrm{kWh}$.

Tabela 63 - Número de clientes por tarifa e horário com Payback em anos, para o gerador diesel.

Tabela 64 - Número de clientes, com TIR maior ou igual a 15\%, por grupo tarifário e tarifa para o gerador diesel.

Tabela 65 - Número de clientes por tarifa e horário com Payback em anos, para o gerador a gás natural.

Tabela 66 - Número de clientes, com TIR maior ou igual a 15\%, por grupo tarifário e tarifa para o gerador a gás natural.

Tabela 67 - Mercado deslocado da LIGHT por tarifa considerando a microturbina com o consumo específico de $0,636 \mathrm{~N} \mathrm{~m}^{3} / \mathrm{kWh}$.

Tabela 68 - Mercado deslocado da LIGHT por tarifa considerando a microturbina com o consumo específico de $0,440 \mathrm{~N} \mathrm{~m}^{3} / \mathrm{kWh} . \quad 194$

Tabela 69 - Mercado deslocado da LIGHT por tarifa para o gerador diesel. 194 Tabela 70 - Mercado deslocado da LIGHT por tarifa para o gerador GN. 194

Tabela A.1 - Desempenho na produção de Potência.

Tabela A.2 - Condições do GN, fluido recuperador de calor (água) e gases de exaustão da planta de cogeração da PUC-Rio.

Tabela A.3 - Desempenho na produção de Potência e Calor.

Tabela A.4 - Valores da relação ar - combustível, vazão de ar, vazão de combustível e vazão dos gases de exaustão.

Tabela B.1 - Desempenho na produção de Potência.

Tabela B.2 - Condições do GN, fluido recuperador de calor (água) e gases de exaustão da planta de cogeração da PUC-Rio.

Tabela B.3 - Desempenho na produção de Potência e Calor.

Tabela B.4 - Valores da relação ar - combustível, vazão de ar, vazão de combustível e vazão dos gases de exaustão.

Tabela C.1 - Desempenho na produção de Potência.

Tabela C.2 - Condições do GN, fluido recuperador de calor (água) e gases de exaustão da planta de cogeração da PUC-Rio.

Tabela C.3 - Desempenho na produção de Potência e Calor. 
combustível e vazão dos gases de exaustão.

Tabela D.1 - Desempenho na produção de Potência. 231

Tabela D.2 - Condições do GN, fluido recuperador de calor (água) e gases de exaustão da planta de cogeração da PUC-Rio.

Tabela D.3 - Desempenho na produção de Potência e Calor.

Tabela D.4 - Valores da relação ar - combustível, vazão de ar, vazão de combustível e vazão dos gases de exaustão.

Tabela E.1 - Desempenho na produção de Potência.

Tabela E.2 - Condições do GN, fluido recuperador de calor (água) e gases de exaustão da planta de cogeração da PUC-Rio.

Tabela E.3 - Desempenho na produção de Potência e Calor.

Tabela E.4 - Valores da relação ar - combustível, vazão de ar, vazão de combustível e vazão dos gases de exaustão.

Tabela F.1 - Desempenho na produção de Potência.

Tabela F.2 - Condições do GN, fluido recuperador de calor (água) e gases de exaustão da planta de cogeração da PUC-Rio.

Tabela F.3 - Desempenho na produção de Potência e Calor.

Tabela F.4 - Valores da relação ar - combustível, vazão de ar, vazão de combustível e vazão dos gases de exaustão. 


\section{Lista de Abreviaturas, Siglas e Símbolos}

ANEEL - Agencia Nacional de Energia Elétrica

${ }^{\circ} \mathrm{C}$ - Grau Celsius (Unidade no Sistema Internacional para temperatura)

CA - Corrente Alternada

CEG - Companhia Distribuidora de Gás Natural do Rio de Janeiro

DPC - Digital Power Controller (Controlador Digital de Potência)

E.B.P.E.R. - Exhaust Back Pressure Efficiency Ratio

E.B.P.P.R. - Exhaust Back Pressure Power Ratio

GC - Modo de operação "Grid Connect"

CEG - Companhia Distribuidora de Gás Natural do Rio de Janeiro

$c_{p_{\text {gaus }}}$ - Calor específico a pressão constante dos gases de exaustão da microturbina

GD - Geração Descentralizada ou Distribuída de Energia Elétrica

GN - Gás Natural

$\mathrm{Hz}-\mathrm{Hertz}$

INMETRO - Instituto Nacional de Metrologia, Normalização e Qualidade Industrial.

ISO - International Organization for Standardization

LIGHT SESA - Light Serviços de Eletricidade S.A.

LPNG - Low Pressure Natural Gas

mA - Mili ampere

$\dot{m}_{\text {gases }}$ - Vazão mássica dos gases de exaustão da microturbina

MTG - Microturbina à Gás

$\mathrm{PCl}$ - Poder calorífico inferior do combustível

PCS - Poder calorífico superior do combustível.

PósMQI - Programa de Pós-Graduação em Metrologia para Qualidade e Inovação 
PRODIST - Procedimentos de Distribuição de Energia Elétrica no

Sistema Interligado Nacional

PUC-Rio - Pontifícia Universidade Católica do Rio de Janeiro

RFC - Rotary Flow Compressor (compressor rotativo)

RPM - Rotação por minuto

SA - Modo de operação "Stand-Alone"

SEP - Sistema Elétrico de Potência

SI - Sistema Internacional de Unidades

SPV - Smart Proporcional Valve (Válvula Proporcional Inteligente)

$\mathrm{T}_{\mathrm{amb}}$ é a temperatura ambiente

$\mathrm{T}_{\mathrm{ex}}$ - Temperatura de saída dos gases de exaustão da microturbina

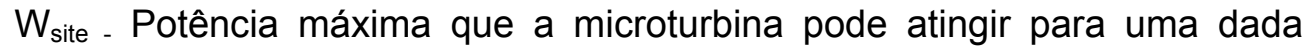
condição ambiental: 


\title{
Símbolos Gregos
}

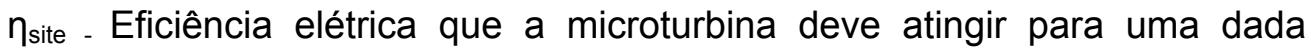 \\ condição ambiental \\ $\eta_{\text {micro }}$ - Eficiência elétrica da microturbina \\ $\Omega-\mathrm{Ohm}$
}

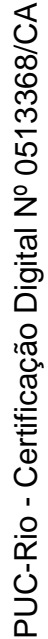

